\title{
Mindfulness, Consciousness and Quantum Physics
}

\author{
Paolo Di Sia \\ University of Padova, School of Engineering, Stradella S. Nicola 3, I-36100 Vicenza (Italy) \\ paolo.disia@libero.it_http://www.paolodisia.com
}

\begin{abstract}
The relation between quantum physics and higher brain functions, including the consciousness, is in recent years a top subject of deep discussions from a scientific, philosophical, psychological and existential point of view. Reality is in continuous motion, life is continuously becoming, a movement of propagating energy waves. The quantum revolution undermined the solid nature of reality, opening the door to the primary role of mind, and proposing scenarios with unexpected features. In this paper interesting aspects of quantum physics are considered, with inevitable repercussions on our lives, which can decisively influence our actions and future. These findings underline the importance of building a cognitive resilience with mindfulness training, for improving the ability to regulate emotions, attention to the true surrounding reality, mood and well-being in life.
\end{abstract}

Keywords: Consciousness, Entanglement, Mind, Mindfulness, Quantum Physics, Retrocausality, Well-being.

\section{Introduction}

The relation between quantum physics and higher brain functions, including the consciousness, is a very discussed subject in last years, by various points of view. We have from a hand physicists, that try to explain the mind-brain problem in terms of modern physics, on the other cognitive neuroscientists and neurobiologists, that in majority consider the quantum world as not relevant for solving their problems.

Given the enormous computation power of neurons in our brain, can consciousness be explained in a purely neurobiological framework, or is there space for quantum calculus in the brain? Biological organisms are composed of atoms and molecules, therefore they obey both to classical and quantum physics laws, with the nanometric world as intermediate (Di Sia, 2014). Unlike classical physics, quantum physics is probabilistic but, despite its many open conceptual and interpretative problems, it explains a whole series of phenomena that cannot be understood in a classical context. Among the "weird features" of the quantum world, we remember in particular:

a) the "wave-particle duality": light and particles act both as waves (wave aspect) and as particles (particle aspect), depending by the experimental configuration;

b) the "indetermination principle of Heisenberg": the impossibility of precisely determining both the position and the velocity of a quantum object, having always a margin of approximation, even if very small;

c) the phenomenon of "entanglement": two or more objects can be highly correlated even if they are separated by great distance, and the behavior of one of them "instantly" influences the behavior of the others, violating our ideas about the concept of "locality" and the fact that the speed of light, currently known as the maximum reachable speed, is finite (in empty space it is 299792,458 kilometers per second). Entanglement is one of the most difficult "paradoxes" of quantum theory, because it implies an "action at distance" without 
any intermediation. Frontier's experiments could open the way for the application of entanglement also to macroscopic systems, i.e. to objects of the daily world (Di Sia, 2017).

Quantum theory, despite its problems in particular related to the role of the conscious observer in the act of measurement, has solved a lot of questions of classical physics and has opened the door to new and interesting technological unthinkable applications. Being classical physics an approximation of quantum one, everything should in fact be described with this last; but many scientists consider the brain only as a classical object. One of the crucial questions concerns the possibility that all components of the nervous system, a "strongly coupled fabric" to its environment and "living" at ordinary environment temperature, show quantum macroscopic behaviors, such as quantum entanglement, that are also connected to the "problem of consciousness" (Di Sia \& Licata, 2016).

The quantum revolution has undermined the solid and tangible nature of reality. Scientists have been faced with three inseparably linked mysteries:

a) the nature of the universe;

b) the nature of consciousness;

c) the origin of universe and consciousness.

A recent developing scientific sector is concerned with the connection between quantum aspects and psychology, with all implications for living a fully aware life. Let's talk about "quantum psychology", "well-being", "lifespan training" for adults, youngs and children, through personal education and channeled into mainstreams, such as the school path.

\section{Brain and quantum computation}

The brain is an extremely complex reality, probably the most complex known by human being; complex quantum systems are notoriously difficult to analyze, except for highlyidealized models or "at the limit" models. It is well known that estimations based on the same pattern for one particle, applied to millions and millions of interacting particles, show discrepancies of various orders of magnitude. For this reason people use computational theories for getting neural correlations of quantum processes in the brain (Di Sia, 2014; Nielsen \& Chuang, 2002).

Quantum computation is not easy to implement; it tries to use the entanglement by checking that the system converges with strong probability on the result. In its simplest version, a quantum computer transforms the initial state of many qubits (which are the quantum version of classical bits) by preserving the probability, through a sequence of quantum logic gates, externally controllable, in a final state with a result of probabilistic nature.

There is the problem of "noise" in computing, the so-called "decoherence", always present, although partially cancelled by particular techniques. The big drawbacks of quantum computing seem to paint a rather unhappy picture for its application in the brain; for example, the pre- and post-synaptic receptors and other components at the basis of neuronal excitability are so "big" that they can be treated as classical objects.

One of the most famous proposals for quantum physics in the brain is the "ORCH-OR" model performed by Roger Penrose and Stuart Hameroff. One of the main issues that divide scientists is the consciousness: is it to be understood as a simple sub-product of information processing processes (and therefore in principle reproducible also on a computer), or derives by peculiar characteristics of the brain (Hameroff \& Penrose, 2014)?

According to Penrose, consciousness would be the product of quantum-type probabilistic effects. His thesis has been criticized both at a scientific and philosophical level, 
since the brain is considered by a part of the scientific community to be unsuitable for quantum effects. These criticisms should be reviewed considering recent discoveries of various mechanisms, such as the sense of smell and the photosynthesis, which appear to be influenced by quantum mechanics.

According to this model, consciousness would be based on quantum vibrations in microtubules within brain neurons; these vibrations have been actually confirmed by observations in the brain. The quantum vibrations of microtubules can be related to some electroencephalographic rhythms that have not been explained otherwise, as proof of their influence on brain processes.

Two fundamental biophysical operations are the basis of processing information in the brain: the chemical transmission through the synaptic slice and the generation of action potentials. They include thousands of neurotransmitter ions and molecules, coupled in such a way to be extended for dozens of micrometers. According to the conventional neuronal processing, both processes destroy coherent quantum states, implying that neurons can only receive and send classical information.

Many computational mathematical operations are currently available for neurons related to changes in synaptic weights, pre-synaptic activity, dendrites; this has not yet led to a definitive understanding of the brain work, but many neuroscientists believe that there is no need of quantum aspects, that quantum algorithms (which are much more powerful than conventional classical algorithms) are not implemented in the nervous system (Koch, 2004).

\section{The "problem" of consciousness}

Roger Penrose argued that the brain is able to evaluate non-computable functions and that this ability is connected to consciousness; this requires a theory that has been not yet discovered, but would be related to what he has studied over years. The content of consciousness is rich and highly differentiated, is associated with the activity of a very large number of neurons widespread throughout the cortex. It remains to solve the problem of quantum coherence in the millimeters and centimeters of separation among single neurons, to allow quantum information to be "not destroyed", and if consciousness is strictly necessary to the collapse of the wavefunction (Di Sia, 2017).

The empirical demonstration of "weakly decoherent and controllable quantum bits" in neurons connected by electrical or chemical synapses, or the discovery of an efficient quantum algorithm for calculations performed by the brain, would be one of the major tests in favour of quantum physics in the brain.

Consciousness is an evanescent reality, cannot be seen or touched, it is not quantifiable. This is a "principle" problem for science, because it uses the fundamental rule of "measurability" of what it studies and tries to explain. Just as "dark matter" and "dark energy" are hypotheses for overcoming great problems of consistency of currently used physical models, some researchers have also suggested that consciousness could be considered as a "new state of matter".

The hypothesis has been presented for first time in 2014 by the theoretical physicist Max Tegmark; he suggested that there is a new state of matter, just like solid, liquid, and aeriform states, in which atoms process information, give rise to subjectivity and, ultimately, to consciousness. He proposed the name "perceptronium" for this new state of matter (Tegmark, 2015).

The supported argument starts from the following assumptions: generations of physicists and chemists studied what happens when a large number of atoms meets, finding that their collective behavior depends on how they are disposed. The key difference between a 
solid, a liquid or a gas is not in the type of considered atoms, but in their disposition. According to Tegmark, there would be no particular physical areas of perceptronium in the brain (that moving in blood leads to a sense of self-awareness), rather the consciousness could be interpreted as result of a particular set of mathematical conditions. Various forms of consciousness might arise just as conditions for the creation of different states of matter (such as steam, water and ice). It is necessary to understand what is needed to produce these different states of consciousness, according to observable and measurable conditions.

This idea has been inspired by the work of neuroscientist Giulio Tononi, who in 2008 proposed the "Integrated Information Theory" (IIT), in which he indicated possible ways to highlight the characteristics of consciousness:

a) a conscious being must be able to store, process and recall large amounts of information;

b) this information must be integrated into a unified set, so that it is impossible to divide it into independent parts (Tononi, Boly, Massimini, \& Koch, 2016).

This means that consciousness must be considered as a whole and cannot be divided into separate components. A human being or a conscious system must therefore not only be able to store and process information, but must do it in a way that forms a complete and indivisible set. He introduced also a mathematical quantity $\phi$ that could be used to measure the "level of consciousness" of a system.

Subsequently Tegmark suggested that there are two types of matter which could be considered in accordance with Tononi's integrated information theory:

a) the "computronium", which meets the requirements of the first aspect, i.e. to be able to store, process and recall large amounts of information;

b) the "perceptronium", which in addition realizes the modality of the "indivisible whole" of Tononi.

He has also identified five fundamental principles that could be used to distinguish conscious matter from other types of matter such as solids, liquids and gases: "information, integration, independence, dynamics, utility principles". He also tried to clarify how his new way of thinking about consciousness could explain the unique human perspective in the universe.

\section{The retrocausal quantum theory}

Lately theoretical physics provided new support to the argument that, if reasonable assumptions are made, quantum theory must be retrocausal. This does not mean that signals can be communicated by the future to the past (considering thermodynamic reasons and not considering tachyonic particles); retrocausality means that when a person chooses the measurement setting for doing a measurement, for example a measure on a particle, his decision may affect the properties of that particle (or another particle) in the past, i.e. $a$ decision taken in the present may affect something in the past.

To explain observations on particles far apart, each of which seems to "immediately" know the made measurement on the other one, the only valid explanation has been the "action at distance" (entanglement). But allowing the possibility that the measurement of particle could retroactively influence the behavior of other particles, there would be no need of the action at distance, but only of a "retroactive effect" (Leifer \& Pusey, 2017).

Huw Price, one of the major supporter of retrocausality in quantum mechanics, provided an argument suggesting that any quantum theory which assumes that:

a) the quantum state is real; 
b) the quantum world is temporally symmetrical: the physical processes described by the same physical laws can be performed back and forth, i.e. substituting " $t$ " with "- $t$ " in the equations of motion, must allow retroactive effects. Since temporal symmetry seems to be a fundamental physical symmetry, it is argued that it is more reasonable to allow retrocausality (Knee, 2017).

Knee created an algorithm to design optimal experiments providing a strong test that quantum state is an ontic state (a state of reality) and not an epistemic state (a state of knowledge). If retrocausality is a feature of the quantum world, then it would have enormous implications for understanding the foundations of quantum theory, proving that quantum theory is incomplete and retrocausality can be one of the missing pieces that complete it.

\section{Mind, Mindfulness, Will of Well-Being and Positivity}

Can our thoughts change the course of events and change our reality? It is possible to get a different reality from what we are if we start to change the way we think. If the way of thinking does not change, then the emotions do not change and consequently do not change the filter used to codify the reality, so the future reality will be the same as the lived one. Only a conscious act can break this chain and allow us to generate different thoughts with respect to the past ones. To change our reality, we need to change our thoughts and emotions, transforming them to what we now aren't but we want to be. We need to be "visionaries, dreamers" (Emerson, 2016).

The thoughts we formulate are consistent with our past experiences. The past determines our way of thinking. We must make a dive in the past, repeat the experiences and eliminate negative polarities. We need to be today what we want to be tomorrow, not to think about what we want, but to become what we want. What matters is the "adopted mentality" to deal with life and the mentality is something that only we can change, in our favor or not. We need to learn art and science for cultivate types of conscious experiences that promote wellbeing and cultivate resilience, for a global health improvement (Tanzi \& Chopra, 2013).

The mind includes consciousness and information processing, as well as a regulatory function called "self-organization". It can be trained to influence our thoughts, emotions and body. Human awareness and respect are at the center of well-being, strengthen the courage, joy, and inner peace. Awareness allows the reduction of pathologies and increases positive psychological and physiological states, the fully being at this moment at multiple levels, i.e. physically, emotionally, cognitively, relationally, and spiritually. "Being present" promotes growth and well-being.

Long-term stress damages telomeres, degrades cognitive functioning and weakens the ability to regulate emotions, but only when it is not counteracted by resilience factors. Positive states of mind seem to increase the telomerase enzyme and maintain telomeres over years. Although the most popular treatments for depression are today the use of antidepressants, current research is showing how awareness, compassion and some other key mentalities can equally be powerful in causing the release of natural antidepressants in the brain.

The gradual cognitive decline is a normal aspect of aging. Also non-genetic factors have a big impact on the health and well-being of the brain. A correct lifestyle can minimize the risk factors of cognitive decline, including physical conditioning, through stress reduction methods, proper nutrition and mental exercises, creating a mentality that transforms healthy brain behaviors into lifestyle habits which can optimize the well-being throughout the lifespan (Chopra, 2015; Chopra \& Kafatos, 2017). 


\section{Conclusions}

Quantum mechanics opened the door to the primary role of mind and how we should not look only at the "visible reality", but that there is much more. There is still no real knowledge of what consciousness is, but we must also consider what is outside the realm of human beings. If consciousness is an emerging feature of a highly integrated network, probably all complex systems (not necessarily only human beings) might have a minimum form of consciousness, even if qualitatively different by the human one.

Many aspects of quantum physics can be relevant for the practice of psychotherapy, as well as the identification of common elements between psychoanalysis and quantum physics. All that helps us to understand what consciousness really is, how it works and what characteristics it has. These are new and extremely interesting intersections, that could change the face of physics, neurology, psychology and many other fields.

Everyday the life puts us in front to choices and decisions, that over time become more and more difficult considering their consequences on our actions and future. It is imperative, in order to set a life on positivity and well-being, to hard work on will and action by building cognitive resilience with the formation of awareness.

Deliberately the paper did not take into account the Heidegger's position about the "being-in-the-world", understood as an inquiry into the world's idea as such is, as a fundamental condition of human existence, the way we "feel home in the world", regardless of any further occupation and activity.

It should not be considered an error the crossed investigation of various levels of explanation made in this paper and the idea of an influence of quantum effects on other reality levels whose theoretical and conceptual framework is not directly correlated to quantum world. It has been a desired path, considering that quantum reality is today a pillar of science, with the classic explanations as approximations of the quantum ones, more or less effective depending on the context.

\section{References}

Chopra, D. (2015). Quantum Healing (Revised and Updated): Exploring the Frontiers of Mind/Body Medicine. New York: Bantam Books.

Chopra, D., Kafatos, M C. (2017). You Are the Universe: Discovering Your Cosmic Self and Why It Matters. New York: Harmony Books.

Di Sia, P. (2014). Analytical Nano-Modelling for Neuroscience and Cognitive Science. Journal of Bioinformatics and Intelligent Control, 3(4), 268-272.

Di Sia, P. (2014). Present and Future of Nanotechnologies: Peculiarities, Phenomenology, Theoretical Modelling, Perspectives. Reviews in Theoretical Science, American Scientific Publishers, 2(2), 146-180.

Di Sia, P. (2017). Quantum Physics, Metaphysics, Theism: Interpretations, Ontologies, Theological Remarks. World Scientific News, 74, 106-120.

Di Sia, P., Licata, I. (2016). Nano-Modelling and Computation in Bio and Brain Dynamics. Bioengineering, 3(11), (7 pp), doi:10.3390/bioengineering3020011.

Emerson, R. W. (2016). The Conduct of Life. CreateSpace Independent Publishing Platform (CreateSpace.com).

Hameroff, S., Penrose, R. (2014). Consciousness in the universe: a review of the 'Orch OR' theory. Physics of Life Reviews, 11(1), 39-78. 
Knee, G. C. (2017). Towards optimal experimental tests on the reality of the quantum state. New Journal of Physics, 19, 023004 (10 pp.).

Koch, C. (2004). The Quest for Consciousness: A Neurobiological Approach. Colorado: Roberts.

Leifer, M. S., Pusey, M. F. (2017). Is a time symmetric interpretation of quantum theory possible without retrocausality?. Retrieved (July 14, 2017) from: arXiv:1607.07871 [quant-ph].

Nielsen, M., \& Chuang, I. (2002). Quantum Computation and Quantum Information. Cambridge: Cambridge Univ. Press.

Tanzi, R. E., Chopra, D. (2013). Super Brain: Unleashing the Explosive Power of Your Mind to Maximize Health, Happiness, and Spiritual Well-Being. New York: Three Rivers Press.

Tegmark, M. (2015). Consciousness as a State of Matter. Retrieved (July 14, 2017) from: https://arxiv.org/abs/1401.1219.

Tononi, G., Boly, M., Massimini, M., Koch, C. (2016). Integrated information theory: from consciousness to its physical substrate. Nature Reviews Neuroscience, 17, 450-461. 